\title{
Extracorporeal Shock Wave Therapy Is Effective in the Treatment of Bone Marrow Edema of the Medial Compartment of the Knee: A Comparative Study
}

\author{
Valerio Sansone ${ }^{a, b}$ Pietro Romeo ${ }^{b}$ Vito Lavanga ${ }^{a}$

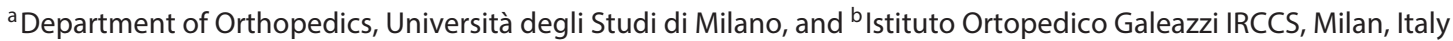

\section{Keywords \\ Bone marrow edema syndrome $\cdot$ Knee $\cdot$ Medial compartment $\cdot$ Conservative treatment $\cdot$ Extracorporeal shock waves · Magnetic resonance imaging}

\begin{abstract}
Objective: To test the hypothesis that shock wave therapy can produce a statistically significant improvement in symptoms and imaging features of the knee bone marrow edema syndrome (BMES) within 6 months of treatment. Subjects and Methods: Eighty-six consecutive patients suffering from BMES of the medial compartment of the knee were prescribed a course of high-energy extracorporeal shock wave therapy (ESWT) and clinically followed up at 3 and 6 months and finally from 14 to approximately 18 months after treatment. Thirty-one patients were unable to undergo ESWT but returned for the 6-month and final follow-up; these were referred to as the conservative (control) group, while the other 55 patients constituted the ESWT group. The Western Ontario and McMaster Universities Arthritis Index (WOMAC) and Visual Analog Scale (VAS) score of each patient were calculated at every follow-up. The BME area was assessed using magnetic resonance imaging before treatment and the 6-month follow-up. Results: Statistically significant improve-
\end{abstract}

\begin{tabular}{ll}
\hline KARGER & ( 2016 S. Karger AG, Basel \\
$\begin{array}{l}\text { E-Mail karger@karger.com } \\
\text { www.karger.com/mpp }\end{array}$ & $\begin{array}{l}\text { This is an Open Access article licensed under the terms of the } \\
\text { Creative Commons Attribution-NonCommercial 3.0 Un- } \\
\text { ported license (CC BY-NC) (www.karger.com/OA-license), } \\
\text { applicable to the online version of the article only. Distribu- } \\
\text { tion permitted for non-commercial purposes only. }\end{array}$
\end{tabular}

ments were observed in clinical scores and in the BME area for both the ESWT and the control group $(p<0.05)$. The improvements in the ESWT group were statistically better in all parameters compared with the control group: the ESWT group had a reduction in the BME area of $86 \%$ versus $41 \%$ in the control group, the VAS pain score improved by $88 \%$ in the ESWT group versus $42 \%$ in the control group, and the WOMAC score improved by $65 \%$ in the ESWT group versus $22 \%$ in the control group. Clinical scores were significantly better for patients with medial tibial lesions in the ESWT group. Conclusion: In this study, ESWT reduced pain and the BME area in the knee, with significant clinical improvement noticed 3 months after treatment.

(c) 2016 S. Karger AG, Basel

\section{Introduction}

Bone marrow edema (BME) is an accumulation of fluid in extracellular marrow spaces, and it is a feature of numerous physiological and pathological states. This reversible, nonspecific condition, which usually spreads from the medullar space into the subchondral region of the joint, appears as an area of ill-defined, homogeneous, intermediate signal intensity on T1-weighted (T1W) images 
and high signal intensity on fat-suppressed T2-weighted (T2W), proton density, or short-time inversion recovery pulse sequences [1]. Pathological BME is related to various causes, and, although the exact pathogenetic processes still remain unknown $[2,3]$, it is most likely a vascular reaction to external or internal disorders. Reflecting its compound etiology, BME usually displays different extension, localization, and signal intensity, and, for this reason, the term subchondral bone marrow lesions would be preferred [4]. Although there is still debate over the association of the BME syndrome (BMES) with other diseases such as aseptic osteonecrosis, trabecular microfractures, and algodystrophy, it is now an accepted clinical entity.

The BMES usually affects the epiphyses of weightbearing joints; most frequently the hip, but also the knee, foot, and ankle, although it may also manifest itself as a "migratory" BME with multiple episodes in different locations [1]. In the knee, it is seen in response to various causes: ischemia (osteochondritis dissecans, osteonecrosis, or complex regional pain syndrome), mechanical causes (bone contusion or bone bruises, microfractures, or stress fractures), and in response to other causes (osteoarthritis, surgery, or cancer) [5]. Symptoms are disabling and impair the quality of life and efficiency of this mainly working-aged, active patient population. The natural history indicates that relief from clinical symptoms and normalization of magnetic resonance imaging (MRI) require 3-18 months [3]. However, some authors support the hypothesis that it may represent an early form of avascular necrosis [5].

A gold standard for the treatment of BMES of the knee does not exist to date. Owing to the usually favorable and self-limiting prognosis, conservative treatment based on protected weight-bearing, analgesic, and physical therapies is recommended, although surgical treatment with bone core decompression has also been reported [6]. Recently, the use of intravenous prostacyclin and bisphosphonates has also been described [7].

Among the physical therapies, extracorporeal shock waves (ESW) have proven to be very effective in the treatment of musculoskeletal disorders due to their angiogenic and trophic effects on tissue [8]. More specifically, clinical trials have highlighted the efficacy of ESW therapy (ESWT) in treating the early stages of avascular necrosis of the femoral head, where a decrease in the extension of bone edema and related pain was observed [9]. Similar results, in terms of pain reduction, functional improvement, and normalization of MRI features, have been observed with ESWT of BMES of the hip [8], and of BMES accompanying Kienböck disease of the wrist [10].
Consequently, we hypothesized that ESWT might improve symptoms in BMES of the medial compartment of the knee (medial femoral condyle and medial tibial plateau). The choice to study exclusively the medial compartment was made in order to reduce variables; the knee joint has a complex architecture due to the presence of 3 compartments (medial, lateral, and patellofemoral); each of the compartments presents 2 articular sides and each is a potential site of BME. Moreover, the medial compartment is the most frequent location of knee BME [11]. Therefore, we performed a study to test the hypothesis that ESWT could result in a statistically significant improvement in clinical symptoms and imaging features of BMES affecting the medial compartment of the knee within 6 months of treatment.

\section{Patients and Methods}

From January to December 2012, 88 consecutive patients (32 males, 56 females) suffering from symptomatic BME of the medial compartment of the knee joint were recruited to participate in this study. Written informed consent was obtained from each patient, and the study was approved by the institutional review board.

The inclusion criterion was the presence of acute knee pain associated with a bone marrow lesion, defined as a bone high-intensity signal area on $\mathrm{T} 2 \mathrm{~W}$ sequences. The exclusion criteria were: BME with any MRI finding of avascular necrosis, defined as a crescent area in the subchondral bone (low-intensity signal subchondral area on T1W sequences); advanced osteoarthritis of the knee (Ahlbäck grade 3 or 4); BME involving also the lateral compartment and/or femoropatellar joint; systemic conditions such as rheumatoid arthritis, autoimmune diseases, or tumors; previous treatments for the index condition; or presence of contraindications for ESWT. Two female patients were excluded from the cohort (one was pregnant, and the other had been treated previously with bisphosphonates). Hence, of the original 88 patients who presented to our clinic, 86 were included in our study.

Treatment consisted of 1 session of shock wave therapy every 3 weeks for 9 weeks ( 3 times in total) using a shock wave electromagnetic source (Epos Ultra Lithotripter; Dornier MedTech $\mathrm{GmbH}$, Wessling, Germany) fitted with an echographic outline pointing device. At each treatment session, 2,000 shots were applied at high energy, with energy flux density ranging from 0.22 to $0.43 \mathrm{~mJ} / \mathrm{mm}^{2}$ and a frequency of $4 \mathrm{~Hz}$.

Protected weight bearing ( 2 crutches) was prescribed as long as pain was present, and analgesics were given on demand. Restriction of physical activity was also recommended, whereas cycling and swimming were encouraged, if tolerated.

All patients were clinically evaluated by the same single examiner (V.L.), blinded to the MRI results, according to the Western Ontario and McMaster Universities Arthritis Index (WOMAC) 100 -point scale, which included pain, ability to walk unaided, autonomy in daily activities, and range of motion. Patients were additionally asked to rate their pain level on a 10-point Visual Analog 
Table 1. Characteristics of the patients in the extracorporeal shock wave therapy group (ESWT) and the conservative therapy group (control)

\begin{tabular}{lll}
\hline & $\begin{array}{l}\text { ESWT } \\
(n=55)\end{array}$ & $\begin{array}{l}\text { Control } \\
(n=31)\end{array}$ \\
\hline BMI & $26.3 \pm 3.8$ & $25.7 \pm 3.6$ \\
Age, years & $59.8 \pm 11.7$ & $61.1 \pm 13.71$ \\
Time from symptom onset to treatment initiation, weeks & $6.9 \pm 7.3$ & $6.2 \pm 8.1$ \\
Gender & $33(60 \%)$ & $21(67.7 \%)$ \\
$\quad$ Female & $22(40 \%)$ & $10(32.3 \%)$ \\
$\quad$ Male & $27(49.1 \%)$ & $17(54.8 \%)$ \\
$\quad \begin{array}{l}\text { Medial femoral condyle } \\
\quad \text { Medial tibial plateau }\end{array}$ & $28(50.9 \%)$ & $14(45.2 \%)$ \\
\hline
\end{tabular}

One male patient in the control group did not present at the final follow-up.

Scale (VAS), where 0 represented no pain, and 10 the maximum possible or unbearable pain. These scores were assessed before and 3 and 6 months after treatment. A final follow-up to obtain data for this study was performed approximately 18 months after treatment. The patients were weighed $(\mathrm{kg})$, height was measured $(\mathrm{m})$, and body mass index (BMI) was then calculated $\left(\mathrm{kg} / \mathrm{m}^{2}\right)$. The age of each patient and the period of time patients had been suffering from BMES were noted.

All the patients also underwent a knee MRI examination before and 6 months after treatment. An experienced radiologist examined all the MR images on the same MRI monitor. The presence of subchondral focal low-intensity signal areas - a possible expression of early osteonecrosis - was evaluated and excluded on T1W sequences. Then, bone marrow lesion areas were evaluated on fatsuppressed fast spin echo T2W sequences. A quantitative measurement of the edema area (in $\mathrm{mm}^{2}$ ) was performed using the Osirix MD Software (Pixmeo SARL) and obtained by measuring the area on the same sagittal and coronal plane slices of the MRI scans before and after treatment.

Thirty-one patients who lived far from our hospital were unable to find a suitable center to undergo the shock wave therapy. They were advised to follow the conservative protocol previously published [1] consisting of protected weight bearing and analgesics for pain control. Non-weight bearing was prescribed until the acute pain reduced, and then a single crutch was used until full weight bearing did not produce discomfort. Cycling and swimming were encouraged within the limits of pain. These 31 patients underwent a clinical and radiological follow-up approximately 6 months after their first visit as well as a further clinical follow-up to provide data for this study at approximately 18 months. These patients constituted the conservative (control) group and the other 55 patients were treated with ESWT.

Both control and ESWT groups were further subdivided by localization of the BME (medial femoral condyle and medial tibial plateau). The clinical and imaging results of the treatment and the control group, and of the femoral condyle and the tibial plateau subgroup, were analyzed and compared. One male patient in the control group did not present at the final follow-up.

\section{Statistical Analysis}

Means and SD were calculated for the BME area on MR images before and 6 months after treatment, and for the clinical scores before treatment and at the final follow-up, and means and SD were calculated for each of the outcome measures at the various time points. The results of the two groups, and of the subgroups (femoral condyle and tibial plateau), were compared using a $t$ test for differences in means, with the level of significance set at $p<$ 0.05 . The population study was tested for normal distribution before the $t$ test was applied.

\section{Results}

The first follow-up visit for the control group was at $6.2 \pm 1.7$ months (mean \pm SD); the final follow-up for the ESWT group was at $19.3 \pm 1.98$ months and for the control group at $19.1+2.01$ months. There were no significant differences between the mean BMI, age, or time between onset of symptoms and treatment of the study group compared with the control group (31 patients; $p<$ 0.05; Table 1).

There were no statistical differences between the mean values of the two groups in any of the measured parameters before treatment: WOMAC, $p=0.41$; VAS, $p=0.53$; and BME area, $p=0.65$. For all the patients, both in the treatment and the control group, there were statistically significant improvements in all parameters measured over the course of the study ( $p<0.001$; Table 2$)$. However, the improvements were significantly greater for the ESWT group: the mean WOMAC score improved by 34.9 points $(65 \%)$ in the treated group, whilst that of the comparison group was 11.6 points $(22 \%$; $p<0.001)$. Likewise, 
Fig. 1. MR images of a patient treated with shock wave therapy. The fat-suppressed fast spin echo T2W MR images show the reduction in the hyperintense signal of the subchondral tibial plateau bone (arrows) from before treatment to the 6-month follow-up in the coronal plane $(\mathbf{a}, \mathbf{b})$ and the sagittal plane $(\mathbf{c}, \mathbf{d})$.
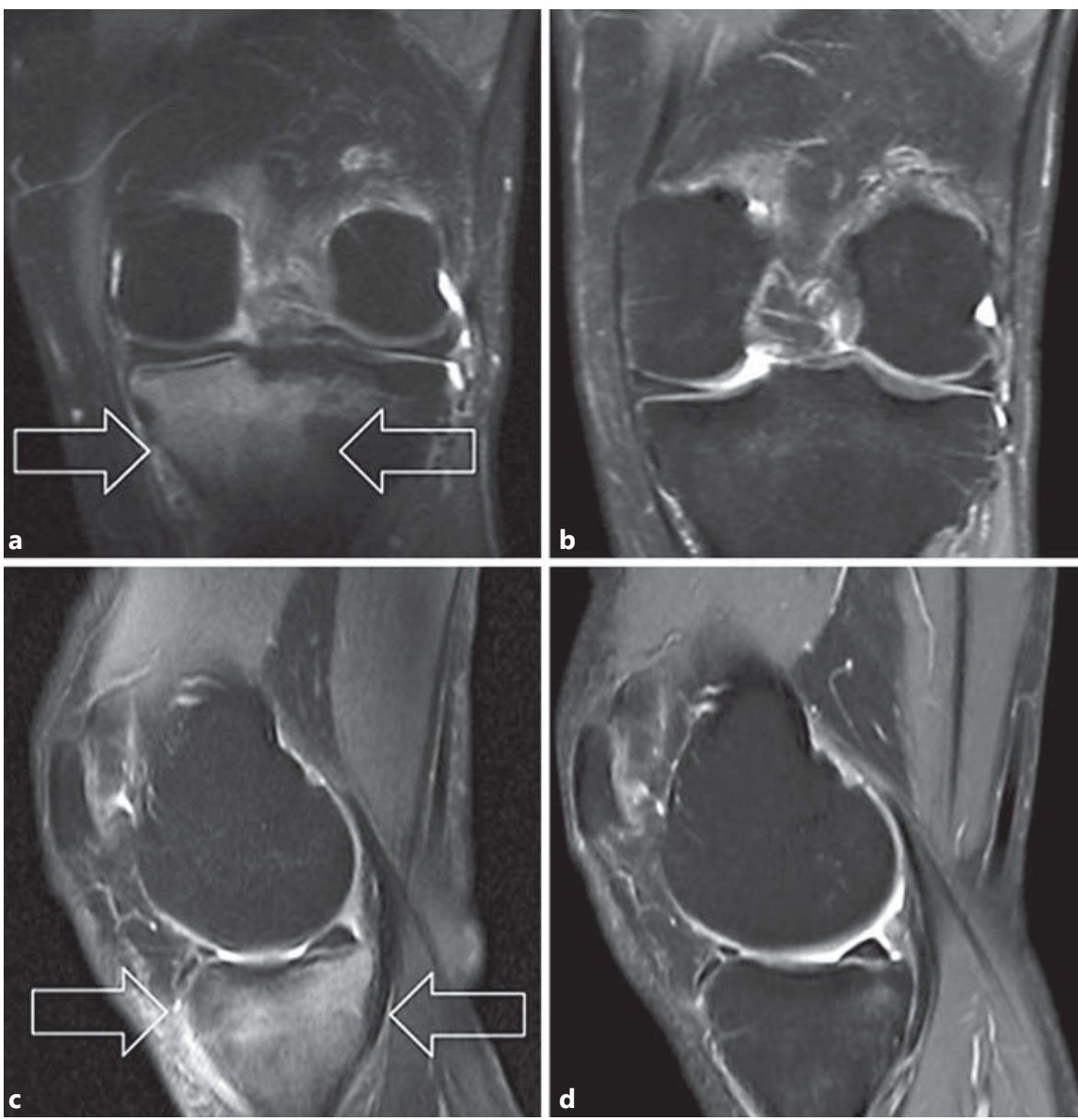

the mean VAS score improved $6.9(88 \%)$ points in the ESWT group (indeed 29 [53\%] patients were completely pain free), whereas in the control group VAS score improvement was $3.2(42 \%)$, with no pain-free patient $(p<$ 0.001 ). In the ESWT group, 11 patients had VAS scores over 2 (mean: $3.7 \pm 0.91$ points; range $2.5-5$ ) at the final follow-up, although in all these cases the area of edema on MRI had reduced by at least $70 \%$.

The MRI findings demonstrated BME regression in most of the treated patients (Fig. 1); indeed, by 6 months after ESWT, the mean BME area had reduced by $88 \%$ compared to before treatment $(p<0.001$; Table 2$)$. The $\mathrm{BME}$ regression in the control group was less marked (41\% reduction), although it was still statistically significant $(p<0.001)$, and in 4 patients $(13 \%)$ the edema had completely resolved.

The clinical and imaging results based on the site of the BME are given in Tables 3 and 4 . The improvement in clinical results was significantly better for patients with tibial lesions (WOMAC score: $p=0.04$, VAS score: $p=$ 0.02 ), but the reduction in the BME area did not significantly differ between femoral and tibial lesions $(p=$ 0.054). Comparisons by lesion site between the ESWT and the control group showed that the improvements in all parameters (VAS, WOMAC, and edema area) both for the tibial and the femoral lesions were significantly better for the ESWT group than the control group $(p<0.001)$.

All the patients who underwent ESWT $(n=55)$ tolerated the treatment well, and no side effects were reported.

\section{Discussion}

The most important finding of the present study was the ability of ESWT to significantly reduce pain and improve functionality in all patients by 3 months after treatment. At this point, all patients had already regained a significant level of autonomy in their daily activities with 
Table 2. MRI and clinical observations throughout the study period using extracorporeal shock wave $(n=55)$ and conservative therapy (control)

\begin{tabular}{lllll}
\hline & Before treatment & 3 months & 6 months & Final follow-up \\
\hline WOMAC score & & & & \\
ESWT $(n=55)$ & $53.55 \pm 10.92$ & $87.43 \pm 7.5$ & $88.39 \pm 6.8$ & $88.27 \pm 6.92$ \\
Control $(n=31)$ & $51.93 \pm 8.84$ & N/A & $62.39 \pm 6.94$ & $63.56 \pm 7.48$ \\
\hline VAS score & & & & \\
ESWT $(n=55)$ & $7.84 \pm 1.27$ & $1.58 \pm 2.08$ & $1.49 \pm 1.01$ & $0.9 \pm 1.27$ \\
Control $(n=31)$ & $7.70 \pm 0.95$ & N/A & $4.76 \pm 1.26$ & $4.52 \pm 1.19$ \\
\hline BME area on $M$ MRI, $\mathrm{mm}^{2}$ & & & & \\
ESWT $(n=55)$ & $759.98 \pm 291.39$ & N/A & $88.38 \pm 131.32$ & N/A \\
Control $(n=31)$ & $739.69 \pm 139.03$ & N/A & $430.49 \pm 157.74$ & N/A \\
\hline
\end{tabular}

ESWT, extracorporeal shock wave therapy; WOMAC, Western Ontario and McMaster Universities Arthritis Index; VAS, Visual Analog Scale; BME, bone marrow edema; MRI, magnetic resonance imaging.

Table 3. MRI and clinical observations throughout the study period for patients with lesions in the medial femoral condyle

\begin{tabular}{lllll}
\hline & Before treatment & 3 months & 6 months & Final follow-up \\
\hline WOMAC score & & & & \\
ESWT $(n=27)$ & $53.47 \pm 12.11$ & $85.95 \pm 7.33$ & $86.11 \pm 7.3$ & $86.04 \pm 7.59$ \\
Control $(n=17)$ & $51.93 \pm 8.84$ & N/A & $62.39 \pm 6.94$ & $63.56 \pm 7.48$ \\
\hline VAS score & & & & \\
ESWT $(n=27)$ & $7.81 \pm 1.33$ & $1.49 \pm 1.87$ & $1.26 \pm 1.91$ & $1.35 \pm 1.5$ \\
Control $(n=17)$ & $7.70 \pm 0.95$ & N/A & $4.76 \pm 1.26$ & $4.52 \pm 1.19$ \\
\hline BME area on $M R I, m^{2}$ & & & $162.35 \pm 145.59$ & N/A \\
ESWT $(n=27)$ & $804.94 \pm 292.22$ & N/A & $418.36 \pm 160.1$ & N/A \\
Control $(n=17)$ & $752.74 \pm 152.15$ & N/A & & \\
\hline
\end{tabular}

WOMAC, Western Ontario and McMaster Universities Arthritis Index; ESWT, extracorporeal shock wave therapy; VAS, Visual Analog Scale; BME, bone marrow edema; MRI, magnetic resonance imaging.

Table 4. MRI and clinical observations throughout the study period for patients with lesions in the medial tibial plateau

\begin{tabular}{lllll}
\hline & Before treatment & 3 months & 6 months & Final follow-up \\
\hline WOMAC score & & & & \\
ESWT $(n=28)$ & $53.65 \pm 9.54$ & $89.24 \pm 7.47$ & $91.18 \pm 4.98$ & $91.1 \pm 4.9$ \\
Control $(n=14)$ & $51.52 \pm 7.61$ & N/A & $61.61 \pm 6.84$ & $62.27 \pm 7.48$ \\
\hline VAS score & & & \\
ESWT $(n=28)$ & $7.86 \pm 1.23$ & $1.02 \pm 1.31$ & $0.6 \pm 1.01$ & $0.39 \pm 0.67$ \\
Control $(n=14)$ & $7.72 \pm 0.93$ & N/A & $5.23 \pm 1.08$ & $5.07 \pm 0.87$ \\
BME area on $M$ MRI, $\mathrm{mm}^{2}$ & & & & \\
ESWT $(n=28)$ & $711.11 \pm 288.87$ & N/A & $24.83 \pm 49.95$ & N/A \\
Control $(n=14)$ & $721.53 \pm 119.31$ & N/A & $447.38 \pm 156.36$ & N/A \\
\hline
\end{tabular}

WOMAC, Western Ontario and McMaster Universities Arthritis Index; ESWT, extracorporeal shock wave therapy; VAS, Visual Analog Scale; BME, bone marrow edema; MRI, magnetic resonance imaging. 
a marked reduction in pain. From 3 to 6 months after treatment, the improvement in scores was more gradual, but still a statistically significant improvement in WOMAC score was seen at 6 months. These improvements were maintained over the duration of the study. Likewise, there was a highly significant reduction in the mean VAS scores over the course of the study, with the greatest reduction in pain seen at the first follow-up.

These findings support the outcome of previous experience where ESWT was used for the treatment of BMES of the hip, in which a significant and quick reduction in pain and in the area of BME on MRI was observed [8]. Further, these findings compare favorably with the surgical and pharmacological therapies [6] that had been adopted for treating idiopathic BMES of the knee. Baier et al. [7] reported a reduction in the BME area of $50 \%$ and a complete regression in $30 \%$ of all cases at the 1 -year of follow-up with MRI after the use of prostacyclin or bisphosphonates (ibandronate) [7]. Kraenzlin et al. [12] reported that bisphosphonate treatment was able to produce a rapid improvement in clinical symptoms and disability, with complete $(53 \%)$ or partial $(21 \%)$ resolution of pain. Berger et al. [6] reported good results with surgical core decompression of the femoral condyle in 18 patients affected by BMES of the knee. All patients clinically recovered within 6 weeks, and MRI findings normalized within 12 weeks after surgery. Open wedge osteotomy of the tibia has also been proposed in the treatment of BME accompanying early-stage osteoarthritis with medial knee pain [13]. At the 12th-month follow-up, BME had disappeared in $80 \%$ of the treated patients. Although the results observed in this study compare extremely favorably with the other treatments described, it is important to note that ESWT is a simple, noninvasive treatment without significant side effects, whereas adverse effects are reported for the above-mentioned drugs [14], and there are obvious risks and costs accompanying any surgery.

The absence of a validated treatment algorithm reflects the lack of information about the pathogenesis and the clinical implications of BME, particularly regarding the real nature of this condition. Specifically, there is no consensus as to whether it may be considered a benign, selflimiting disease or if it may progress to a more serious state of avascular necrosis of the bone. As has recently been described in the treatment of BMES of the hip [8], the rationale for the usage of ESWT to treat BME arises from the results of recent basic research and clinical studies about the effect of ESWT on musculoskeletal tissues and on conditions that have clinical features similar to BMES of the knee.
Experimental investigations suggested that ESW exert an angiogenic and trophic effect on musculoskeletal tissues [15]. In bone tissue, this response is mediated by specific molecules like vascular endothelial growth factor, proliferating cell nuclear antigen, bone morphogenetic proteins (e.g., BMP-2), and endothelial nitric oxide (NO) synthase [16]. Of greatest interest is the role of endothelial NO synthase, which induces the production of NO, a vasoactive agent produced by endothelial resident cells.

ESW also stimulate osteoblasts and periosteal cells and induce osteogenic differentiation of mesenchymal stem cells $[17,18]$ and an increased production of bone deposition markers, like osteocalcin and C-terminal procollagen type I [19]. In addition, shock waves seem to play a direct role on bone modeling and remodeling by stimulating the proliferation and the differentiation of osteoblasts and, at the same time, through a reduction in proosteoclastogenic factors [19]. Finally, experimental models demonstrate that, in healthy bone, unfocused shock waves positively affect the bone microarchitecture by enhancing the mass and the strength of the tissue, whereas in osteoporotic bone they reduce the bone loss [20].

The clinical application of ESW in bone has given remarkable results in the nonunion of fractures and in the treatment of osteonecrosis of the femoral head, where ESWT significantly reduces the extension of both necrotic area and BME surrounding the ischemic lesion; a significant reduction in the associated pain has also been reported [9].

On the basis of this scientific evidence and from the results of previous investigations $[8,10]$, we continued the clinical study of the effects of ESW in vascular and metabolic disorders of the bone. As previously postulated for hip BMES, it is supposed that ESWT may normalize the mechanisms that govern bone homeostasis in BMES of the knee. The primary objective in the management of this condition is the relief of pain, but treatments should also aim to avoid the weakening of the subchondral bone trabeculae, which results from an altered bone turnover in the presence of edema [21], and to prevent a possible progression to osteonecrosis [1].

In the knee, BME lesions in the tibial plateau responded better to the shock wave treatment than those in the femoral condyle; the improvements in clinical scores for the tibia were significantly better, and a greater reduction in the mean BME area was also observed. In our opinion, this result may be explained by local differences in the blood supply to anatomic areas of the knee. This could also be a reason for the propensity of BME to affect the medial femoral condyle. 
Although the BME area on MRI scans had not disappeared in all cases, the functional results were all good or excellent. It has been reported that the BME area is not necessarily correlated to pain [22], and that MRI abnormalities may take up to 16 months before resolving [23].

The control group was actually treated mainly with weight-bearing restrictions, although there is no conclusive evidence to suggest that weight bearing as tolerated will adversely affect the outcome. The positive results observed also in this group are a demonstration of the substantially benign nature of the disease and favorably compare with the reports of several authors in the literature $[1,24]$.

The limitations of this study are the lack of a randomized control group consisting of nontreated symptomatic patients (however, it would have been unethical to refuse treatment to a symptomatic patient) and the inability to ascertain whether or not the edema area had continued to reduce over the full duration of the study, as an MRI examination was not performed at the final follow-up.

\section{Conclusion}

In this study, ESWT produced rapid pain relief and functional improvement within 3 months after treatment, and could aid in the normalization of the vascular and metabolic impairments which characterize BMES. Nevertheless, further controlled studies with different treatment protocols in terms of number and intervals of treatments, number of shots, and the energy level employed are needed to establish the optimal regimen.

\section{Acknowledgments}

The author would like to thank Alberto Zerbi for his technical assistance.

\section{References}

1 Hofmann S, Kramer J, Vakil-Adli A, et al: Painful bone marrow edema of the knee: differential diagnosis and therapeutic concepts. Orthop Clin North Am 2004;35:321-333.

2 Hofmann S, Kramer J, Breitenseher M, et al: Bone marrow edema in the knee. Differential diagnosis and therapeutic possibilities (in German). Orthopäde 2006;35:463-475.

3 Meizer R, Radda C, Stolz G, et al: MRI-controlled analysis of 104 patients with painful bone marrow edema in different joint localizations treated with the prostacyclin analogue iloprost. Wien Klin Wochenschr 2005; 117:278-286.

4 Hofmann S: The painful bone marrow edema syndrome of the hip joint. Wien Klin Wochenschr 2005;117:111-120.

5 Hofmann S, Kramer J, Schneider W, et al: Transient osteoporosis may represent a reversible early form of avascular necrosis of the hip joint. Curr Orthop 1997;11:164-172.

6 Berger CE, Kröner AH, Kristen KH, et al: Transient bone marrow edema syndrome of the knee: clinical and magnetic resonance imaging results at 5 years after core decompression. Arthroscopy 2006;22:866-871.

7 Baier C, Schaumburger J, Goetz J, et al: Bisphosphonates or prostacyclin in the treatment of bone-marrow oedema syndrome of the knee and foot. Rheumatol Int 2013;33: 1397-1402.

8 D'Agostino C, Romeo P, Lavanga V, et al: Effectiveness of extracorporeal shock wave therapy in bone marrow edema syndrome of the hip. Rheumatol Int 2014;34:1513-1518.
9 Wang CJ, Wang FS, Huang CC, et al: Treatment for osteonecrosis of the femoral head: comparison of extracorporeal shock waves with core decompression and bone-grafting. J Bone Joint Surg Am 2005;87:2380-2387.

10 D'Agostino C, Romeo P, Amelio E, et al: Effectiveness of ESWT in the treatment of Kienböck's disease. Ultrasound Med Biol 2011;37: 1452-1456.

11 Pape D, Seil R, Fritsch E, et al: Prevalence of spontaneous osteonecrosis of the medial femoral condyle in elderly patients. Knee Surg Sports Traumatol Arthrosc 2002;10:233-240.

12 Kraenzlin ME, Graf C, Meier C, et al: Possible beneficial effect of bisphosphonates in osteonecrosis of the knee. Knee Surg Sports Traumatol Arthrosc 2010;18:1638-1644.

13 Kesemenli CC, Memisoglu K, Muezzinoglu US, et al: Treatment for painful bone marrow edema by open wedge tibial osteotomy. Eur J Orthop Surg Traumatol 2013;23:825-829.

14 Assaf AT, Smeets R, Riecke B, et al: Incidence of bisphosphonate-related osteonecrosis of the jaw in consideration of primary diseases and concomitant therapies. Anticancer Res 2013;33:3917-3924.

15 Sansone V, D’Agostino MC, Bonora C, et al: Early angiogenic response to shock waves in a three-dimensional model of human microvascular endothelial cell culture (HMEC-1). J Biol Regul Homeost Agents 2012;26:29-37.
16 Wang CJ, Yang KD, Wang FS, et al: Shock wave therapy enhances bone mass and bone strength after fracture of the femur. A study in rabbits. Bone 2004;34:225.

17 Wang FS, Yang KD, Kuo YR, et al: Temporal and spatial expression of bone morphogenetic proteins in extracorporeal shock wave-promoted healing of segmental defect. Bone 2003;32:387-396.

18 Martini L, Giavaresi G, Fini M, et al: Effect of extracorporeal shock wave therapy on osteoblastlike cells. Clin Orthop Relat Res 2003; 413:269-280.

19 Tamma R, Dell'Endice S, Notarnicola A, et al Extracorporeal shock waves stimulate osteoblastic activities. Ultrasound Med Biol 2009; 35:2093-2100.

20 Van Der Jagt OP, Van Der Linden JC, Shaden $\mathrm{W}$, et al: Unfocused extracorporeal shock wave therapy as potential treatment for osteoporosis. J Orthop Res 2009;27:1528-1533.

21 Vande Berg BC, Malghem JJ, Lecouvet FE, et al: Idiopathic bone marrow edema lesions of the femoral head: predictive value of MR imaging findings. Radiology 1999;212:527-535.

22 Unay K, Poyanli O, Akan K, et al: The relationship between bone marrow edema size and knee pain. Knee Surg Sports Traumatol Arthrosc 2009;17:1298-1304.

23 Boks SS, Vroegindeweij D, Koes BW, et al: Follow-up of occult bone lesions detected at MR imaging: systematic review. Radiology 2006;238:853-862.

24 Vardi G, Turner PJ: Transient osteoporosis of the knee. Knee 2004;11:219-223. 